\title{
Phospholipase A2 as a novel therapeutic target in lung cancer
}

\author{
Fang-Yuan Zhang ${ }^{1,2 *}$, Run-Ze Li ${ }^{1,2 *}$, Jia-Xin Li ${ }^{1,2}$, Xing-Xing Fan ${ }^{1,2}$, Chun- Xie ${ }^{1,2}$, Liang Liu ${ }^{1}$, Xiao-Jun Yao ${ }^{1,2 *}$ and Elaine Lai-Han Leung ${ }^{1-3 *}$ \\ ${ }^{1}$ Dr. Neher's Biophysics Laboratory for Innovative Drug Discovery, Macau University of Science and Technology, Avenida Wai Long, China \\ ${ }^{2}$ State Key Laboratory of Quality Research in Chinese Medicine/Macau Institute for Applied Research in Medicine and Health, Macau University of Science and \\ Technology, China \\ ${ }^{3}$ Zhuhai Hospital of Traditional Chinese and Western Medicine, P.R. China
}

\begin{abstract}
Secreted PLA2 (sPLA2) enzyme, which shows the unique cellular properties and distinct biological roles, is the lipolytic enzyme that acts on glycerophospholipids to induce the release of free fatty acids (FAs). Many researches have reported the human sPLA2s are expressed abnormally in various cancers. But it is still not clear about the specific isoforms function of sPLA2 in these cancers, and its function roles in various cancers. In this review, we will introduce the mechanism and pathway of sPLA2 in cancers as well as the relationship with lipid metabolism. Then we will further discuss the novel technologies which can be applied to explore the action of particular sPLA2 enzyme in lung cancer and the mechanisms of drugs such as small molecule inhibitors or traditional Chinese medicines, with the potential to treat diseases associated with inflammation and lipid metabolism.
\end{abstract}

\section{Role and pathogenesis of SPLA2 on cancer}

Phospholipase A2 (PLA2) isthe various class of esterase that cleaves glycerophospholipids at the sn-2 bond to yield the fatty acid and the lysophospholipid [1]. PLA2 has more than 30 isoforms, which was divided into six subfamilies: cytosolic PLA2 (cPLA2), calciumindependent PLA2 (iPLA2), small molecular weight secretory PLA2 (sPLA2), lysosomal PLA2, platelet activating factor acetylhydrolases, and adipose specific PLA2 (AdPLA2) [2].

PLA2 enzyme has the function to regulate the release of biologically active fatty acids and lysophospholipids from membrane phospholipid pools, suggesting its role as substrates for intracellular biochemical pathways that generate potent autocrine and paracrine lipid mediators participating in regulating cell proliferation, survival, differentiation, motility, tissue vascularization [3,4]. cPLA2 $\alpha$ (the prototypic cPLA2) plays a major role in the initiation of arachidonic acid metabolism, the iPLA2 family contributes to membrane homeostasis and energy metabolism, and the sPLA2 family generally function outside of the cell [5]. Although there are a lot of researches focused on the sPLA2, the specific isoform enzyme of sPLA2 in lung cancer especially sPLA2 is still unclear. In this review, we will highlight and mianly talk about the function and key role of sPLAs in cancer.

It has been reported that PLA2 subtypes (IIA, IID, IIF, III, V, X) are expressed in different cancers, while the mechanism has not been elaborated clearly yet. As indicated in (Table 1), the pathological meachnism of sPLA2s in cancer is associated with arachidonic acid (AA) metabolism and stimulation of AA synthesis [6-8]. AA is released from the sn-2 position of phospholipids and serves as a substrate for intracellular biochemial pathways generating over hundred potent autocrine and paracrine lipid mediators, which can be transformed into eicosanoids through various downstream enzymes to modulate the tumour progression such as cell growth, apoptosis, migration and inflammatory processes.
As shown in (Figure 1), sPLA2 induces the release of AA and leads to the COX-2-dependent PGE2 formation in cancer cells. The cyclooxygenase (COX) and lipoxygenase (LOX) pathways are implicated in the cancer progress, which inhibitior of COX and LOX pathways could effectively inhibit the development of lung, colon and prostate cancer [6] .

Human secreted phospholipase A2 (HGIIA sPLA2) plays different roles in cancers. HGIIA sPLA2 has been testifeid to increase in patients with lung, oesophageal and prostate cancer, but decreases in gastric cancer $[7,8]$. HGIIA sPLA2 is overexpressed in lung cancer cells and widely regarded as a ligand for the epidermal growth factor receptor (EGFR) with the poential to activate the HER/HER2-elicited signaling [9], as shown in (Figure 1). In addition, sPLA2 inhibitor is capable of decreasing the expression of ICAM-1in human esophageal adenocarcinoma cells, which is induced by nuclear factor- $\mathrm{\kappa B}$ activation [10]. Meanwhile, since the HGIIA sPLA2 controbutes to the prostate tumor progression, it could act as a prognostic biomarker for prostate cancer [11]. Moreover, HGIIA sPLA2 is also dected in colon cancer. However, HGIIA sPLA2 expression appears to reduce in gastric cancer. S100 calcium-binding protein A4 (S100A4) is of great importance in the metastatic progression of tumor cells through impacting on several steps in the metastatic cascade, including migration, invasiveness, and angiogenesis, and is associated with prognosis in several cancers

${ }^{*}$ Correspondence to: Elaine Lai-Han Leung and Xiao-Jun Yao, Department of Thoracic Surgery, Guangzhou Institute of Respiratory Health and State Key Laboratory of Respiratory Disease, The First Affiliated Hospital of Guangzhou Medical University, Guangzhou, P.R. China, Tel: +853-8897-2409, Fax: +8532882-2799, E-mail: 1hleung@must.edu.mo / xjyao@must.edu.mo

Received: December 03, 2020; Accepted: December 22, 2020; Published: December 24, 2020 


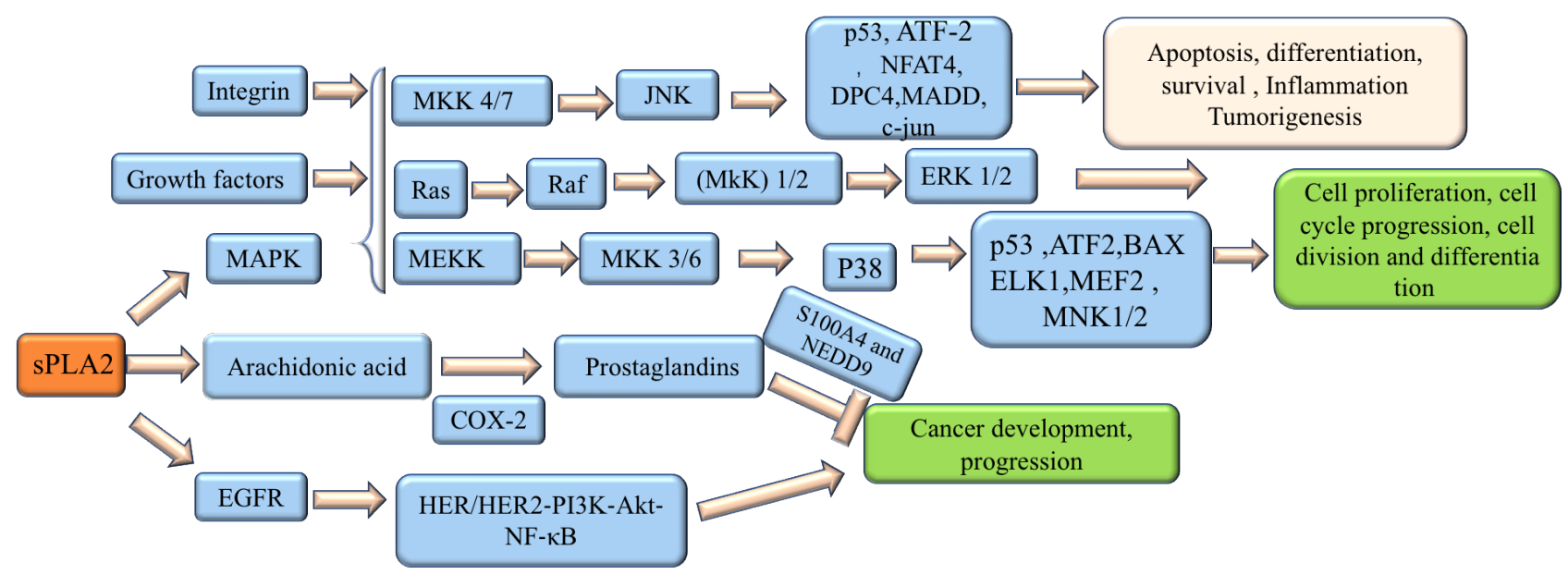

Figure 1: The SPLA2 signaling pathways in cancers

Table 1. The function of SPLA2 subtypes in different cancers

\begin{tabular}{|c|c|c|c|}
\hline Subtypes & Type & Function & Reference \\
\hline \multirow{9}{*}{ II A } & Lung cancer Cells & sPLA2-IIa attenuates growth and promotes apoptosis predominantly via its effects on NF-kB activity. & {$[78]$} \\
\hline & & sPLA2-IIa functions as a ligand for the EGFR family of receptors, elevating HER/HER2-elicited signaling. & [9] \\
\hline & & $\begin{array}{l}\text { Plasma sPLA2-IIa is a potential lung biomarker to distinguish benign nodules from lung cancer and to aid lung cancer } \\
\text { diagnosis in patients with SPNs. }\end{array}$ & [79] \\
\hline & Prostate cancer & $\begin{array}{l}\text { sPLA2-IIa could serve as a prognostic biomarker for prostate cancer and a potential surrogate prostate biomarker indicative of } \\
\text { tumor burden. }\end{array}$ & {$[11]$} \\
\hline & & $\begin{array}{l}\text { sPLA2-IIa expression is inversely related to } 5 \text {-year patient survival, and inhibitors of the group IIa sPLA2 is promosing for } \\
\text { prostate cancer chemotherapy. }\end{array}$ & {$[80]$} \\
\hline & Stem cells & $\begin{array}{l}\text { Overexpression of sPLA2-IIA leads to depletion of HFSCs and differentiation associated with altered histone modification, } \\
\text { suggesting its prospective clinical implications. }\end{array}$ & {$[81]$} \\
\hline & Astrocytoma cells & $\begin{array}{l}\text { Adipokine leptin is related to the bad prognosis of tumoral and neurodegenerative processes, becoming a potential mediator of } \\
\text { some obesity-related medical complications. }\end{array}$ & {$[82]$} \\
\hline & Gastric cancer & PLA2G2A expression is an independent predictor of survival for patients with gastric cancer. & [76] \\
\hline & Colon cancer & sPLA2 type IIA and X are present in human colon cancer. & [83] \\
\hline \multirow[t]{3}{*}{ IID } & Inflammation and Cancer & $\begin{array}{l}\text { sPLA2-IID acts as an immunosuppressive sPLA2 that allows the microenvironmental lipid balance toward an anti- } \\
\text { inflammatory state, exerting beneficial or detrimental impact depending upon distinct pathophysiological contexts in } \\
\text { inflammation and cancer. }\end{array}$ & {$[84]$} \\
\hline & Ovrian cancer & $\begin{array}{l}\text { sPLA2-IID could act as a prognostic biomarkers and correlats with immune infiltration in ovarian cancer by integrating tumor } \\
\text { mutation burden (TMB) and microenvironment analysis. }\end{array}$ & [85] \\
\hline & Lung cancer & PLA2G2D-Ser enhances the expression of IL-6 and IL-8 compared with PLA2G2D-Gly in A549 cells. & {$[86]$} \\
\hline IIF & Inflammatory process & sPLA 2-IIF is a potent regulator of AA metabolism and participates in the inflammatory process under certain conditions. & [87] \\
\hline \multirow[t]{2}{*}{ III } & Colon cancer & sPLA 2 -III is a good candidate as a novel biomarker for both left and right colon cancers. & {$[54]$} \\
\hline & Colorectal cancer & sPLA 2 -III acts as a novel druggable target for colorectal diseases. & [88] \\
\hline \multirow[t]{2}{*}{ V } & Glioma tumor & PLA2G5 could be a potential biomarker for predicting poor prognosis in patients with gliomas. & {$[51]$} \\
\hline & Cervical cancer & FGF19 and PLA2G5 could be a biomarker to predict patient overall survival in cervical cancer. & [89] \\
\hline \multirow{4}{*}{$\mathrm{X}$} & Colon cancer & HGX sPLA2 promotes cancer cell proliferation and releases various lipid mediators. & {$[90]$} \\
\hline & Breast cancer & HGX sPLA2 promotes breast cancer cell growth and survival by stimulating LD formation and FA oxidation. & [43] \\
\hline & Melanocytes & SPLA2-X plays an important role in cutaneous pigmentation through the release of LPC. & [91] \\
\hline & Breast cancer & PLA2G10 expression is dependent on the subtype of breast cancer. & [92] \\
\hline
\end{tabular}

[12]. Neural precursor cell expressed developmentally down-regulated protein 9(NEDD9) also contributes to the invasion in different kinds of cancers including cervical, lung, melanoma, breast cancer [13-16]. It has been confirmed that HGIIA sPLA2 could inhibit cell invasion and metastasis through regulating S100A4 and NEDD9 gene expression [17]. Hence HGIIA sPLA2 expression can act as an independent predictor of survival for gastric cancer patients, and seems to be a symbol standing for better prognosis, with the potential to become a target for gastric cancer treatment in the future. In addition, HGIIA sPLA2 acts as an antioncogene in intestinal cancer, as the cosmic transgence overexpressing induce the reduction in tumor multiplicity and tumor size [18].

\section{Downstream signaling transduction regulation of SPLA2}

As shown in (Figure 1), HGIIA sPLA2 also modulates mitogenactivated protein kinase (MAPK) signaling [19]. MAPK consists of three distinct signaling pathways: the extracellular signal-regulated protein kinases (ERKs), the c-Jun N-terminal kinases or stressactivated protein kinases (JNK/SAPK), and the p38 family of kinases [20], which are involved in cell functions including proliferation, gene expression, differentiation, mitosis, cell survival, and apoptosis [20]. JNK exerts pivotal roles in inflammation and homeostasis, acting oncogenic or suppressive role in different cancers [21]. JNK-regulated transcription factors help to regulate gene expression in response to a 
variety of cellular stimuli, including stress events, growth factors and cytokines, which activates downstream targets including c-Jun, ATF2, Elk-1, Myc, Smad3, p53, NFAT4, DPC4 and MADD [20]. C-Jun is considered as a cooperating oncogene that has the ablity to induce the cell proliferation, diferentiation and apoptosis. P53, a tumoursuppressor protein exerting several bioloigical functions including regulating metabolism, fecundity, cell differentiation and apoptosis [22]. Moreover, c-Jun has been reported to inhibit p53 expression at the transcriptional level through acting on the $\mathrm{p} 53$ promoter [23]. Ras, one of the GTPases family which are molecular switches that control many signal transduction pathways cellular processes in all eukaryotic cells $[24,25]$ including actin cytoskeleton, membrane transport cell-cycle and cell adhesion [26]. It has been testified that Ras has the ability to induce the activation of Raf [27]. Raf contributes to activate MAPK/ERK kinase (MEK) 1 and MEK2 which in turns catalyze the activation of the ERK1 and ERK2. After activation, ERK1/ERK2 broadly phosphorylates several nuclear effector genes involved in diverse cellular responses such as cell growth, division, migration and angiogenesis. Besides, ERKs could also be activated by several receptor tyrosine kinases and growth factor receptors. MEKK could induce the activation of MKK3/6 kinases,which in turns activates P38. P38 regulates the expression of key inflammatory mediators like cyclooxygenase 2 (COX2), which has been demonstrated to highly expressed in non-melanoma skin cancer, intestinal ,colorectal,cervical and breast cancer [28,29], suggesting P38 may act as a cancer promoter. The other proteins regulated by P38 includes p53, ATF2, Bax, ELK1, MEF2, MNK1/2 [30]. In additon, p38 also participates in several cellular responses such as inflammation, apoptosis, cell cycle and migration.

Hence, there are increasing small-molecule p38 MAPK inhibitors that have undergone clinical trials for their anti-inflammatory and anti-cancer pharmacological action.However, many of them have failed due to the toxicity and adverse side effects $[31,32]$. To sum up, HGIIA sPLA2 participates in the development of cancer by various enzyme-dependent mechanisms.

\section{Basic research on SPLA2 and its metabolic function}

Changes in lipid metabolism can affect numerous cellular and biological processes, and SPLA2s have the ability to influence lipid metabolism [33]. Fatty acid (FA), an important component of lipid, consists of hydrocarbon chains terminating with carboxylic acid groups participating in physiological activity such as energy storage, protein modification, signal-transduction pathways and the generation of signaling molecules. Moreover, as FAs is crucial to tumor progess, limiting the supplies of FAs can decrease the proliferation of cancer cells [34]. FAs also act as the ligands for peroxisome proliferator-activated receptors (PPARs) with three subtypes including $\operatorname{PPAR} \alpha, \operatorname{PPAR} \delta / \beta$, and PPAR $\gamma$. PPARs are ligand-regulated transcription factors, constructing a lipid signaling network between the cell surface and the nucleus as well as regaulating lipid metabolism [35]. PPARa is found to be overexpressed in the heart, liver, kidney and muscle and has the ablility to induce the catabolism of FAs. Moreover, PPAR $\alpha$ expression induces the oxidative stress, cell proliferation and apoptosis inhibition, leading to the increase of hepatocarcinogenesis in mice [36]. To sum up, PPARa exerts a critical role in the metabolism of fatty acid [37,38]. PPAR $\delta$ is involved in the progress of colorectal and colon cancer, being a downstream target gene of APC/ $\beta$-catenin/TCF pathway and Ras/Raf/ MAPK pathway[39]. PPAR $\gamma$ has been demonstrated to be expressed in many kinds of human cancers including lung ,gastric ,prostate ,breast and colon cancer, resulting in the modulation of apoptosis , cell cycle and differentiation [40,41]. PPAR also has inhibitory effect on inflammatory reactions through acting on macrophages, dendritic cells and lymphocytes, which has the potential to treat Inflammation and metabolic disorders.

FAs regulate SREBP-1c expression by mediating its competition with LXR ligand [42]. Meanwhile, FAs released by sPLA2 membrane hydrolysis are incorporated into TAGs and stored in cytosolic LDs, providing a long-term resistance to nutrient and growth factor starvation-induced cell death [43]. The sPLA2-induced LD formation in breast cancer cells is accompanied by the activation of AMPactivated protein kinase (AMPK), maintaining the energy balance by regulating nutrient supply and demands. Previous study has demonstrated that AMPK could decrease DVL3 expression which is related to the activation of the $\mathrm{Wnt} / \beta$-catenin signaling pathway and cell proliferaton in breast cancer. Moreover, it has been testified that the reduction of DVL3 leads to inhibiton of tumor progress in breast cancer. These results show AMPK may act as a therapeutic target in breast cancer [44].

In short, besides SPLA2s is essential in the tumor proliferation and progression, sPLA2s also act as integrated regulators of immune and metabolic responses. However, up to now, there is few study in vivo investigating the promoting tumor mechanism and few research targeting specific subtype in lung cancer. At this moment, there is still no available targed medicine on the market. Thus we propose further research on lung cancer patients based on in vitro, in vivo study and clinical patients validation are worth for investigation in the future.

In biological research, animal models are commonly used to evaluate the growth of tumor. Considering the in vivo microenvironment of primary lung cancer is discriminative from xenograft tumor growth, in sPLA2 research the mice models need to be selected. The several usual mice models used in cancer research include chemically-induced model, xenograft, orthotropic model and human transgenic mice. As xenograft model by hypodermic injection is under dispute recently for the reason that it could not reflect the organ environment of the tumor [45], which is the most regularly-used mouse model to invetigate the function of sPLA2 in vitro. Besides, sPLA2-IIA is the most studied target among the sPLA2 subtypes in cancers. Chemically-induced model is a relatively natural way of tumor induction compared to xenograft, but it takes a long time to induce the formation of tumor [45]. Moreover the chemical used will have influence on metabolites, which cannot be applied in metabolic enzyme study. The transgenic mouse model has advantages in providing a platform to study the tumorigenesis in human, PLA2-IIA transgenic mice has been used to evaluate the functional effects of sPLA2-IIA induced abnormal signalling on normal stem cells and cancer cells [46]. However, the disadvantage is that it is also time-consuming and needs lots of labors [47]. The orthotropic model provides a more natural environment than the xenograft model. Most importantly, we find that orthotropic model can mimic influence on lipid metabolism than xenograft model according to our previous assay. Hence, we recommend to choose the orthotropic model as the main mice model to detect the xenograft growth and changes of metabolomics after specific metabolizing enzyme knockdown. In addition, microCT, magnetic resonance imaging (MRI), near-infrared (NIR) and fluorescence reflectance imaging (FRI) also can be applied to monitor the dynamic growth of tumor in vivo.

\section{Clinical studies of sPLA2}

Overexpression of sPLA2 subtypes correlates with many pathological diseases including arthritis, inflammatory and cardiovascular diseases and many types of cancers, which is always 
related to poor prognosis of cancer patients. Hence sPLA2s have the potential to become therapeutic targets in cancers [48]. As it appears numerous subtypes of sPLA2, the primary difficulty is to identify the specific isoform which functions as a promotor in NSCLC. The common methods to investigate the expression of each sPLA2 isoform in cancer include gene sequencing analysis and protein conventional microarray. With these methods, we could receive clear distinguished isoforms from the samples that we tested. Then depending on other biological assays, such as knockdown and overexpression of the specific isoform gene sequence, we can understand the exact function of each isoform. However, two aspects of disadvantages limit its broad prospect of utilization in different enzymes.

(1). The experimental period is long. Besides the reason for individual differences, it needs a large sample size which makes the time consumption even longer.

(2). The cost is also so high that not every research group could afford it. Thus we paid attention to The Cancer Genome Atlas (TCGA) that is a public funded project aiming to catalogue and discover major cancer-causing genome alterations in human tumours through highthroughput technologies and next-generation genome sequencing for improving the methods of prevention, diagnosis and treatment of cancer $[49,50]$. TCGA has characterized the molecular landscape of tumors from 11,160 patients across 33 cancer types [49]. Comparing to the above mentioned methods, the advantages of TCGA are that it has a larger size of sample collection with little cost and it is easier to get and analyze clinical information and molecular data. Moreover, TCGA could be also used to investigate the connection between the expressions of the certain gene with prognosis of cancers. Since there are lots of researches about the functions or mechnasims of sPLA2 subtypes in human tumors, some studys refer to the connection between expression of the sPLA2 subtypes and cancer prognosis by the utilization of TCGA database. For example, it has reported that phospholipase A2 group V(PLA2G) 5 gene was linked to poor prognosis in patients with gliomas [51]. Therefore, it is feasible and promising to apply the TCGA database to screen the sPLA2 subtypes associated with specific cancer.

Immunohistochemistry (IHC) is a common laboratory tool based on the principle of specific binding of antibody and antigen to detect the distribution and localization of biomarkers and evulate the changes of the protein expression in pathological tissue, which could used for diagnosis and prognostic of cancers [52]. IHC also exerts an important role in pathology, particularly in surgical pathology oncologic pathology, neuropathology [53] .sPLA2s ((IIA, IID, III, V) have been confirmed to elvated in pathological tissue sections from gastric ,prostate, adenocarcinomas and glioma tumors and may acts as biomarkers $[11,51,54,55]$. Hence, we sought to expore more sPLA2 subtypes expression in clinical tissues from cancer patients.

\section{Drug discoveory of sPLA2 and its association with in- flammation and lipid metabolism}

Inflammatory and lipid metabolism is closely related in many disease, such as diabetes, atherosclerosis and cardiovascular disease. Lipid species could positively and negatively regulate inflammatory responses. Conversely, inflammatory signaling able to affect the lipid metabolism in the liver, adipose tissue, skeletal muscle, and other tissues and organs in diseases [56].

Many studies has reported that traditional Chinese medicines (TCMs) have the potential to moderate lipid metabolism and inflammation through various mechnasims and have therapeutic effect on specific diseases. Hence, increasing effective compounds from TCM have been identified. This part, we focus on some of the compounds derived from TCM on several diseases. Diabetes is usually associated with dysregulation of inflammation and the abnormalities of lipid metabolism. Rhizoma Coptidis is a TCM that have been used to treat diabetes in anceient China for thousand of years. Berberine which is the main active compound extracted from Rhizoma coptidis has been found to have effect on patients with type II diabetes by its antilipogenic and hypoglycemic effect and the ability to inhibiting the cholesterol absorption [57,17,58]. Moreover, berberine could inhibit insulin sensitization, insulin secretion and $\beta$ cells regeneration as well as antioxidant activity in experimental rats [59]. Astragalus polysaccharides (APS) extracted from adix Astragali reduces the endoplasmic reticulum stress and the expression of protein tyrosine phosphatase $1 \mathrm{~B}$ in type 2 diabetic rats [60]. Saponin, a chemically heterogeneous class of steroid and triterpenoid glycosides, which is found in many kinds of TCM, has the function to regulate blood lipids, increase glucose tolerance to maintain normal blood sugar levels [61]. Quercetin, a polyphenolic substance widely found in many plants, has the potential to decrease blood glucose levels and elevate plasma insulin levels. Quercetin also could ameliorate vascular functional and structural derangements caused by diabetes[62]. Ginseng is a wellknown TCM that is widespreadly used in east-asian and is permitted as the dietary supplement in the United States. Ginseng is supposed to be an promsing anti-diabetes agents and Ginsenosides is considered as the main active ingredients of ginseng [63]. Disorder of lipid metabolism and inflammation are the pathogenic factors of cardiovascular diseases which is the leading cause of death worldwide.

TCM could be combined with other mediciation to reduce the cardiovascular complications [64]. Salvia miltiorrhiza Bunge, also known as Danshen, a well-known TCMs, which is used for treating cardiovascular $[6,7]$ and cerebrovascular diseases $[8,9]$.Tan IIA is the major compound extracted from Danshen, Tan IIA has been observed to possess antioxidation [10], anti-inflammatory [11] functions which may depend on inhibition of iNOS gene expression, NO production and cytokine (IL-1 $\beta$, IL-6, and TNF- $\alpha$ ) expression via ER-dependent pathway [65]. Moreover, TCM is capable of reducing blood pressure ,it has been reported that tall gastrodia and Uncaria tomentosa could dramatically improve superoxide dismutase levels and decrease the oxidative stress in hypertensive patient [66].

Since sPLA2 is generally recognized as a key tumor-related protein , suggsting it has the prospect of being a therapeutic target on cancers. Moreover, sPLA2 is also involved in the inflammatory process, which is detected to have high level in patients with inflammatory diseases [67]. Hence it will be of great value to develop the inhibitors of sPLA2 as therapeutic alternatives. Therefore, there are increasing inhibitors of sPLA2 being discovered to have effects on sPLA2 through diverse mechanisms. Table 2 shows the present novel sPLA2 inhibitors development progress. Dabrafenib, a FDA-approved drug, has been reported to inhibit the expression of sPLA2-IIa, suggesting that dabrafenib has the potential to used as an anti-inflammatory drug. AZD2716 acts as a potential therapeutic durg for coronary artery disease, due to the excellent preclinical pharmacokinetic properties, in vivo efficacy and minimized risk for drug-drug interactions. A-002 (varespladib methyl/LY333013/S-3013), an oral prodrug of A-001 (varespladib sodium/LY315920/S-5920), could affect the lipid metabolism and increase plasma paraoxonase activity, HDL levels and HDL protective capacity. Indoxam could block the production of the 
Table 2. The inhibitors of SPLA2

\begin{tabular}{|c|c|c|c|}
\hline sPLA2 inhibitor & Target & Function and Application & Reference \\
\hline (R)-7 (AZD2716) & sPLA2-IIa and sPLA2-X & $\begin{array}{l}\text { AZD2716 has excellent preclinical pharmacokinetic properties, in vivo efficacy and } \\
\text { minimized risk for drug-drug interactions and could acts as a clinical candidate for } \\
\text { the treatment of coronary artery disease. }\end{array}$ & {$[93]$} \\
\hline $\begin{array}{l}\text { A-002 (varespladib methyl/ } \\
\text { LY333013/S-3013) }\end{array}$ & sPLA2 & $\begin{array}{l}\text { Varespladib affects the lipid metabolism and elevates HDL levels and HDL } \\
\text { protective capacity, which could also synergize with statins to decrease lesion size. }\end{array}$ & [94] \\
\hline Indoxam & sPLA2-IIA & $\begin{array}{l}\text { Indoxam could block the production of proinfammatory cytokines and signaling } \\
\text { molecules through PLA2- IIA-independent mechanisms, with the potential to be } \\
\text { applied in therapeutic intervention of the cytokine-related systemic infammatory } \\
\text { response syndrome. }\end{array}$ & {$[95]$} \\
\hline RO061606 & SPLA2 IIA & $\begin{array}{l}\text { RO061606 could reduce allergen-induced airway inflammation and } \\
\text { hyperresponsiveness in asthma mouse model, which may become a novel anti- } \\
\text { asthma drugs. }\end{array}$ & [96] \\
\hline $\begin{array}{l}\text { PX-18 (2-N,N-Bis(oleoyloxyethyl) } \\
\text { amino-1-ethanesulfonic acid) }\end{array}$ & sPLA2 & PX-18 has neuroprotection effect on I/R- induced neuronal damage. & {$[97]$} \\
\hline $\begin{array}{l}\text { 7,7-dimethyleicosadienoic acid } \\
\text { (DEDA) }\end{array}$ & sPLA2 IIA & $\begin{array}{l}\text { DEDA could ameliorate secondary injury by reducing exacerbations from } \\
\text { lipoxidative stress. }\end{array}$ & [98] \\
\hline $\begin{array}{l}\text { Dabrafenib (DAB) } \\
\text { (FDA approved) }\end{array}$ & sPLA2 IIA & Dabrafenib may be used to treat vascular inflammatory diseases. & [99] \\
\hline Indole-2-carboxamides & sPLA2-X & $\begin{array}{l}\text { SPLA2-X may become a potential therapeutic target for the treatment of } \\
\text { cardiovascular diseases. }\end{array}$ & {$[100]$} \\
\hline $\begin{array}{l}\text { Standard aqueous stem bark extract } \\
\text { of Mangifera indica } \mathrm{L} \text {. }\end{array}$ & $\mathrm{sPLA}_{2}$ & $\begin{array}{l}\text { Standard aqueous stem bark extract of Mangifera indica } \mathrm{L} \text { could inhibit } \\
\text { inflammatory activities. }\end{array}$ & {$[101]$} \\
\hline SaPLI $\gamma$ & sPLA2 B, IIA, V and X & SaPLI $\gamma$ has effective anti-inflammatory effects in vivo. & {$[102]$} \\
\hline Varespladib & sPLA2 & $\begin{array}{l}\text { Varespladib has the potential to become an economical and effective path forward } \\
\text { to fill the pre-referral gap in the setting of snakebite. }\end{array}$ & [94] \\
\hline Rhamnetin & sPLA2 & Rhamnetin can be deloveped into a new anti-inflammatory drug. & {$[103]$} \\
\hline
\end{tabular}

proinfammatory cytokines and reduce PLA $\mathrm{A}_{2}$-induced LPC production in native and glycoxidized LDL as well as LDL-associated MCP-1 mRNA expression in HUVECs, with the possible to study for agents against atherosclerotic diseases. Unfortunately, most of them have failed to pass the clincal assay due to the adverse reactions.

\section{Novel technologies advance}

After identifying the functional isoform of sPLA2 in lung cancer, the following question is to investigate whether dysregulationof this enzyme accelerate the tumor cell growth or to enhance cell invasion. According to the sPLA2 pathway, the obvious alteration will be observed in the abnormal phospholipid metabolism biomarker. Except for metabolomics assay, there is a novel technique which can be applied. However, so far, a few papers were published in cancer study. Time-of-flight secondary ion mass spectrometry (ToF- SIMS) is the one for materials characterization and is used for biological sample analysis $[68,69]$, usually testing ion level. For example, ToF- SIMS was applied to detect the metabolic changes in pancreatic $\beta$ cell tumors and the surrounding tissues [70].

The advantages of ToF-SIMS can be covered in following parts. Firstly, it enables the chemical specificity in addition to high spatial resolution imaging of biological samples from cells to tissue [69]. Secondarly, SIMS experiment doesn't need initial separation step, which is different from other instrument shuch as GC-MS, LC-MS and NMR. Thirdly, SIMS analysis dose not need derivatization step, hence the nature of the secondary ions is decided by the sample type and the interaction of the primary ion beam with that sample [71]. The last but not least, due to the capability to generate chemical images of the sample parallelly, the results could present with a more intuitive description. To sum up, TOF - SIMS is essential in SPLA2 analysis. Owing to the ability to localize the abnormally secreting site of the products by sPLA2 in tumor frozen sections and the assistance of principal component analysis (PCA), TOF - SIMS will help us visually learn the distribution of the products, including LPC and LPE, by sPLA2 both in tumor and in organs, such as liver. Therefore, applying TOF - SIMS to identify the abnormal phospholipid metabolism biomarker in plasma from lung cancer patients and healthy people with phospholipid metabolomics technology is considerable. Metabolomics is an effective approach to investigate the development of the cancer. It has been demonstrated that metabolites have changed in clincal samples from cancer patients when comparing to the controls. Increasing metabolic biomarkers associated with tumor indicating that the cancer risk have been already identified with the help of TOF - SIMS or other chromatography and mass spectrometers. Here are several examples:

(1) LysoPC a C18: 0 and PC ae C30:0 could act as cancer biomarkers [72].

(2) 15 differentially expressed metabolites have been found to have the potential for early diagnosis for colorectal cancer [73].

(3) Sarcosine is considered as a potentially important metabolic intermediary of cancer progress through profiling the metabolites from clinical samples of prostate cancer patients [74].

(4) 57 metabolites are idtienfied by electrophoresis time-of-flight mass spectrometry (CE-TOF-MS) on saliva samples from different cancer patients, acting as promising biomarkers on several cancers [75]. Hence, analysis of the serum samples could identify the metabolic features that associated with progress of tumors, since the expression of biomarkers will alter along with the tumor progression. Thus, we should pay attention to the upstream or downstream targets of biomarkers for the further study (sPLA2 subtype). This assay can be used as validation to confirm the results above.

\section{Conclusion and perspectives}

To sum up, sPLA2s may exert either a pro- or anti-tumorigenic role in different cancers through regulating the related enzymatic activity and downstream signaling molecules to influence the tumor cell proliferaton and metastasis. For example, HGIIA sPLA2 is the most studied sPLA2 subtype and has been confirmed to contribute to the tumor progression in many types of cancers such as prostate and lung 
cancer $[9,11]$, but it has cancer inhibition effect on gastric cancer [76], suggesting it could have value to be taken as a target for the treatment for gastric cancer. Small interfering RNAs (siRNAs) with the function of specific gene silencing have the potential to become therapeutic tools. It has been reported that the systemically administered small interfering RNA nanoparticles have the ability to reduce protein and mRNA levels in cancer patients [77]. In additon, sPLA2s have the function to modulate the release of biologically active fatty acids and influence lipid metabolism, acting as integrated regulators of immune and metabolic responses.

But there are still a lot of works and problems to deal with. Firstly, we could apply more novel technologies such as ToF- SIMS to explore metabolism biomarkers associated with tumor development in other types of cancers. Secondly, after the confirmation of this subtype sPLA2 proliferating function in lung cancer, flexible molecular docking and pharmacophore model could be applied to high-throughput screen on the TCM compound library to discover the most effective active ingredients. Thirdly, overexpression of sPLA2 subtypes leads to many pathological diseases such as inflammatory disorder and tumors. Although there appear more and more novel sPLA2 inhibitors which have been demonstrated to have therapeutic effect in in vivo and in vitro assays, few of them have passed the clinical trials. The last but not the least, the integrated functions and mechanisms of sPLA2s in cancers is still not clearly elucidated, which need further research.

\section{Acknowledgements}

This work was supported by the Science and Technology Development Fund, Macau SAR (Grant No: 001/2020/ALC \& 0096/2018/A3 \& 0003/2019/AKP).

\section{Contributions}

(I) Conception and design: Xiao-Jun Yao, Elaine Lai-Han Leung;

(II) Manuscript writing: All authors;

(III) Final approval of manuscript: All authors.

\section{Competing interests}

The authors declare that they have no competing interests.

\section{References:}

1. Cummings BS, Mchowat J, Schnellmann RG (2000) Phospholipase A2s in Cell Injury and Death. J Pharmacol Exp The 294: 793-799. [Crossref]

2. Atsumi G, Murakami M, Kojima K, Hadano A, Tajima M, et al. (2000) Distinct Roles of Two Intracellular Phospholipase A $2 \mathrm{~s}$ in Fatty Acid Release in the Cell Death Pathway. Journal of Biological Chemistry 275. [Crossref]

3. Mchowat J, Creer M (2004) Catalytic Features, Regulation and Function of Myocardial Phospholipase A2. Curr Med Chem Cardiovasc Hematol Agents 2: 209-218. [Crossref]

4. Scott KF, Sajinovic M, Hein J, Nixdorf S, Galettis P, et al. (2010) Emerging roles for phospholipase A2 enzymes in cancer. Biochimie 92: 601-610. [Crossref]

5. Murakami M, Taketomi Y, Miki Y, Sato H, Hirabayashi T, et al. (2011) Recent progress in phospholipase A2 research: From cells to animals to humans. Prog Lipid Res 50: 152-192. [Crossref]

6. Kirschenbaum A, Liu X, Yao S, Levine AC (2001) The role of cyclooxygenase-2 in prostate cancer. Urology 58: 127-131. [Crossref]

7. Menschikowski M, Hagelgans A, Schuler U, Froeschke S, Rosner A, et al. (2013) Plasma Levels of Phospholipase A2-IIA in Patients with Different Types of Malignancies: Prognosis and Association with Inflammatory and Coagulation Biomarkers. Pathol Oncol Res 19: 839-846. [Crossref]

8. Avoranta T, Sundström J, Korkeila E, Syrjänen K, Pyrhönen S, et al. (2010) The expression and distribution of group IIA phospholipase A2 in human colorectal tumours. Virchows Archiv 457: 659-667. [Crossref]
9. Dong Z, Meller J, Succop P, Wang J, Wikenheiser-Brokamp K, et al. (2014) Secretory phospholipase A2-IIa upregulates HER/HER2-elicited signaling in lung cancer cells. Int J Oncol 45: 978-984. [Crossref]

10. Sadaria MR, Meng X, Fullerton DA, Reece TB, Shah RR, et al. (2011) Secretory Phospholipase A2 Inhibition Attenuates Intercellular Adhesion Molecule-1 Expression in Human Esophageal Adenocarcinoma Cells. Ann Thorac Surg 91: 1539-1545. [Crossref]

11. Dong Z, Liu Y, Scott KF, Levin L, Gaitonde K, et al. (2010) Secretory phospholipase A2-IIa is involved in prostate cancer progression and may potentially serve as a biomarker for prostate cancer. Carcinogenesis 31: 1948-1955. [Crossref]

12. Boye K, Mælandsmo GM (2010) S100A4 and metastasis: a small actor playing many roles. Am J Pathol 176: 528-535. [Crossref]

13. Jin Y, Li F, Zheng C, Wang Y, Fang Z, et al. (2014) NEDD9 promotes lung cancer metastasis through epithelial-mesenchymal transition. Int J Cancer 134: 2294-2304. [Crossref]

14. Sima N, Cheng X, Ye F, Ma D, Xie X, et al. (2013) The overexpression of scaffolding protein NEDD9 promotes migration and invasion in cervical cancer via tyrosine phosphorylated FAK and SRC. PLoS One 8: e74594. [Crossref]

15. Kong C, Wang C, Wang L, Ma M, Niu C, et al. (2011) NEDD9 is a positive regulator of epithelial-mesenchymal transition and promotes invasion in aggressive breast cancer. PLoS One 6: e22666. [Crossref]

16. Kim M, Gans JD, Nogueira C, Wang A, Paik J-H, et al. (2006) Comparative oncogenomics identifies NEDD9 as a melanoma metastasis gene. Cell 125: 1269-1281. [Crossref]

17. Ganesan K, Ivanova T, Wu Y, Rajasegaran V, Wu J, et al. (2008) Inhibition of Gastric Cancer Invasion and Metastasis by PLA2G2A, a Novel ?-Catenin/TCF Target Gene. Cancer Res 68: 4277-4286. [Crossref]

18. Cormier RT, Hong KH, Halberg RB, Hawkins TL, Richardson P, et al. (1997) Secretory phospholipase Pla2g2a confers resistance to intestinal tumorigenesis. Nat Genet 17: 88-91. [Crossref]

19. Yu JA, Li H, Meng X, Fullerton DA, Nemenoff RA, et al. (2012) Group IIa secretory phospholipase expression correlates with group IIa secretory phospholipase inhibitionmediated cell death in K-ras mutant lung cancer cells. J Thorac Cardiovasc Surg 144: 1479-1485. [Crossref]

20. Cowan KJ, Storey KB (2003) Mitogen-activated protein kinases: new signaling pathways functioning in cellular responses to environmental stress. J Exp Biol 206: 1107-1115. [Crossref]

21. Wagner EF, Nebreda ÁR (2009) Signal integration by JNK and p38 MAPK pathways in cancer development. Nat Rev Cancer 9: 537-549. [Crossref]

22. Vousden KH, Prives C (2009) Blinded by the Light: The Growing Complexity of p53. Cell 137: 413-431. [Crossref]

23. Leppä S, Bohmann D (1999) Diverse functions of JNK signaling and c-Jun in stress response and apoptosis. Oncogene 18: 6158-6162. [Crossref]

24. Santarpia L, Lippman SM, El-Naggar AK (2012) Targeting the MAPK-RAS-RAF signaling pathway in cancer therapy. Expert Opin Ther Targets 16: 103-119. [Crossref]

25. Etienne-Manneville S, Hall A (2002) Rho GTPases in cell biology. Nature 420: 629635. [Crossref]

26. Bishop AL, Hall A (2000) Rho GTPases and their effector proteins. Biochem $J 348$ 241-255. [Crossref]

27. Santarpia L, Lippman SM , El-Naggar AK (2012) Targeting the MAPK-RAS-RAF signaling pathway in cancer therapy. Expert Opin Ther Targets 16: 103-119. [Crossref]

28. Bachelor MA, Bowden GT (2004) UVA-mediated activation of signaling pathways involved in skin tumor promotion and progression. Semin Cancer Biol 14: 131-138. [Crossref]

29. Timoshenko AV, Chakraborty C, Wagner GF, Lala PK (2006) COX-2-mediated stimulation of the lymphangiogenic factor VEGF-C in human breast cancer. $\mathrm{Br} J$ Cancer 94: 1154-1163. [Crossref]

30. Wagner EF, Nebreda AR (2009) Signal integration by JNK and p38 MAPK pathways in cancer development. Nat Rev Cancer 9: 537-549. [Crossref]

31. Kumar S, Boehm J, Lee JC (2003) p38 MAP kinases: key signalling molecules as therapeutic targets for inflammatory diseases. Nat Rev Drug Discov 2: 717-726. [Crossref] 
32. Yong HY, Koh MS, Moon A (2009) The p38 MAPK inhibitors for the treatment of inflammatory diseases and cancer. Expert Opin Investig Drugs 18: 1893-1905. [Crossref]

33. Santos CR, Schulze A (2012) Lipid metabolism in cancer. FEBS J 279: 2610-2623. [Crossref]

34. Currie E, Schulze A, Zechner R, Walther TC, Farese RV Jr (2013) Cellular Fatty Acid Metabolism and Cancer. Cell Metab 18: 153-1561 [Crossref]

35. Berger J, Moller DE (2002) The Mechanisms of Action of PPARs. Annu Rev Med 53: 409-435. [Crossref]

36. Tachibana K, Yamasaki D, Ishimoto K, Doi T (2008) The Role of PPARs in Cancer. PAR Res 2008: 102737. [Crossref]

37. Fruchart JC (2002) Peroxisome proliferator-activated receptor- $\alpha$ activation and highdensity lipoprotein metabolism. Am J Cardiol 88: 24N-29N. [Crossref]

38. Staels B, Dallongeville J, Auwerx J, Schoonjans K, Leitersdorf E, et al. (1998) Mechanism of Action of Fibrates on Lipid and Lipoprotein Metabolism. Circulation 98: 2088-2093. [Crossref]

39. Shao J, Sheng H, DuBois RN (2002) Peroxisome proliferator-activated receptors modulate K-Ras-mediated transformation of intestinal epithelial cells. Cancer Res 62 : 3282-3288. [Crossref]

40. Tachibana K, Yamasaki D, Ishimoto K, Doi T (2008) The role of PPARs in cancer. PPAR Res 2008: 102737 [Crossref]

41. Sato H, Ishihara S, Kawashima K, Moriyama N, Suetsugu H, et al. (2000) Expression of peroxisome proliferator-activated receptor (PPAR) $\gamma$ in gastric cancer and inhibitory effects of PPAR $\gamma$ agonists. Br J Cancer 83: 1394-1400. [Crossref]

42. Yoshikawa T, Shimano H, Yahagi N, Ide T, Amemiya-Kudo M, et al. (2002) Polyunsaturated Fatty Acids Suppress Sterol Regulatory Elementbinding Protein 1c Promoter Activity by Inhibition of Liver X Receptor (LXR) Binding to LXR Response Elements. J Biol Chem 277: 1705-1711 [Crossref]

43. Pucer A, Brglez V, Payré C, Pungerčar J, Lambeau G, et al. (2013) Group X secreted phospholipase A2 induces lipid droplet formation and prolongs breast cancer cell survival. Mol Cancer 12: 111. [Crossref]

44. Zou YF, Xie CW, Yang SX, Xiong JP (2017) AMPK activators suppress breast cancer cell growth by inhibiting DVL3-facilitated $\mathrm{Wnt} / \beta$-catenin signaling pathway activity. Mol Med Rep 15: 899-907. [Crossref]

45. Wartha K, Herting F, Hasmann M (2014) Fit-for purpose use of mouse models to improve predictivity of cancer therapeutics evaluation. Pharmacol Ther 142: 351-361. [Crossref]

46. Chovatiya GL, Sunkara RR, Roy S, Godbole SR, Waghmare SK (2019) Contextdependent effect of sPLA2-IIA induced proliferation on murine hair follicle stem cells and human epithelial cancer. EBioMedicine 48: 364-376. [Crossref]

47. Rashid OM, Takabe K (2015) Animal models for exploring the pharmacokinetics of breast cancer therapies. Expert Opin Drug Metab Toxicol 11: 221-230. [Crossref]

48. Quach ND (2017) Functions of secreted phospholipase A2 and its interacting proteins in liposome uptake and prostate cancer progression. University of Georgia. [Crossref]

49. Liu J, Lichtenberg T, Hoadley KA, Poisson LM, Lazar AJ, et al. (2018) An integrated TCGA pan-cancer clinical data resource to drive high-quality survival outcome analytics. Cell 173: 400-416.e411. [Crossref]

50. Tomczak K, Czerwińska P, Wiznerowicz M (2015) The Cancer Genome Atlas (TCGA): an immeasurable source of knowledge. Contemp Oncol (Pozn) 19: A68-A77. [Crossref]

51. Wu C, Su J, Wang X, Wang J, Xiao K, et al. (2019) Overexpression of the phospholipase A2 group $\mathrm{V}$ gene in glioma tumors is associated with poor patient prognosis. Cancer Manag Res 11: 3139-3152. [Crossref]

52. Zhu CQ, Shih W, Ling CH, Tsao MS (2006) Immunohistochemical markers of prognosis in non-small cell lung cancer: a review and proposal for a multiphase approach to marker evaluation. J Clin Pathol 59: 790-800. [Crossref]

53. Duraiyan J, Govindarajan R, Kaliyappan K, Palanisamy M (2012) Applications of immunohistochemistry. J Pharm Bioallied Sci 4: S307-S309. [Crossref]

54. Mounier CM, Wendum D, Greenspan E, Flejou JF, Rosenberg DW, et al. (2008) Distinct expression pattern of the full set of secreted phospholipases A 2 in human colorectal adenocarcinomas: sPLA 2-III as a biomarker candidate. Br J Cancer 98: 587-595. [Crossref]
55. Wang X, Huang CJ, Yu GZ, Wang JJ, Wang R, et al. (2013) Expression of group IIA phospholipase A2 is an independent predictor of favorable outcome for patients with gastric cancer. Hum Pathol 44: 2020-2027. [Crossref]

56. Glass CK, Olefsky JM (2012) Inflammation and lipid signaling in the etiology of insulin resistance. Cell Metab 15: 635-645. [Crossref]

57. Ni YX (1988) [Therapeutic effect of berberine on 60 patients with type II diabetes mellitus and experimental research]. Zhong Xi Yi Jie He Za Zhi, 8: 711-713. [Crossref]

58. Wang D, Na Q, Song WW, Song GY (2007) Eulipidemic Effects of Berberine Administered Alone or in Combination with Other Natural Cholesterol-lowering Agents. Arzneimittelforschung 57: 26-30. [Crossref]

59. Zhou J, Zhou S, Tang J, Zhang K, Guang L, et al. (2009) Protective effect of berberine on beta cells in streptozotocin-and high-carbohydrate/high-fat diet-induced diabetic rats. Eur J Pharmacol 606: 262-268. [Crossref]

60. Wang N, Zhang D, Mao X, Zou F, Jin H, et al. (2009) Astragalus polysaccharides decreased the expression of PTP1B through relieving ER stress induced activation of ATF6 in a rat model of type 2 diabetes. Mol Cell Endocrinol 307: 89-98. [Crossref]

61. Zhang T-T, Jiang J-G (2012) Active ingredients of traditional Chinese medicine in the treatment of diabetes and diabetic complications. Expert Opin Investig Drugs 21: 16251642. [Crossref]

62. Mahmoud MF, Hassan NA, El Bassossy HM, Fahmy A (2013) Quercetin protect against diabetes-induced exaggerated vasoconstriction in rats: effect on low grade inflammation. PLoS One 8: e63784. [Crossref]

63. Yuan H-D, Kim JT, Kim SH, Chung SH (2012) Ginseng and diabetes: the evidences from in vitro, animal and human studies. $J$ Ginseng Res 36: 27-39. [Crossref]

64. Li Y, Wang X, Shen Z (2017) Traditional Chinese medicine for lipid metabolism disorders. Am J Transl Res 9: 2038-2049 [Crossref]

65. Fan G-W, Gao X-M, Wang H, Zhu Y, Zhang J, et al. (2009) The anti-inflammatory activities of Tanshinone IIA, an active component of TCM, are mediated by estrogen receptor activation and inhibition of iNOS. J Steroid Biochem Mol Biol 113: 275-280. [Crossref]

66. Hao P-P, Jiang F, Chen Y-G, Yang J, Zhang K, et al. (2015) Traditional Chinese medication for cardiovascular disease. Nat Rev Cardiol 12: 115-122. [Crossref]

67. Grönroos JM, Nevalainen TJ (1992) Increased Concentrations of Synovial-Type Phospholipase $\mathrm{A}<\mathrm{SUB}>2</ \mathrm{SUB}>$ in Serum and Pulmonary and Renal Complications in Acute Pancreatitis. Digestion 52: 232-236. [Crossref]

68. Barreto G, Soininen A, Sillat T, Konttinen YT, Kaivosoja E (2014) Sample processing, protocol, and statistical analysis of the time-of-flight secondary ion mass spectrometry (ToF-SIMS) of protein, cell, and tissue samples. Methods Mol Biol 1142: 177-88 [Crossref]

69. Denbigh J, Lockyer N (2015) ToF-SIMS as a tool for profiling lipids in cancer and other diseases. Materials Science and Technology 31: 137-147. [Crossref]

70. Bluestein BM, Morrish F, Graham DJ, Huang L, Hockenbery D, et al. (2018) Analysis of the Myc-induced pancreatic $\beta$ cell islet tumor microenvironment using imaging ToFSIMS. Biointerphases 13: 06D402. [Crossref]

71. Vickerman J (2012) Evaluating the challenges associated with time-of-fight secondary ion mass spectrometry for metabolomics using pure and mixed metabolites. Metabolomics 9: 535-544. [Crossref]

72. Kühn T, Floegel A, Sookthai D, Johnson T, Rolle-Kampczyk U, et al. (2016) Higher plasma levels of lysophosphatidylcholine 18: 0 are related to a lower risk of common cancers in a prospective metabolomics study. BMC Med 14: 13. [Crossref]

73. Li F, Qin X, Chen H, Qiu L, Guo Y, et al. (2013) Lipid profiling for early diagnosis and progression of colorectal cancer using direct-infusion electrospray ionization Fourier transform ion cyclotron resonance mass spectrometry. Rapid Commun Mass Spectrom 27: 24-34. [Crossref]

74. Sreekumar A, Poisson LM, Rajendiran TM, Khan AP, Cao Q, et al. (2009) Metabolomic profiles delineate potential role for sarcosine in prostate cancer progression. Nature 457: 910-914. [Crossref]

75. Sugimoto M, Wong DT, Hirayama A, Soga T, Tomita M (2010) Capillary electrophoresis mass spectrometry-based saliva metabolomics identified oral, breast and pancreatic cancer-specific profiles. Metabolomics 6: 78-95. [Crossref]

76. Wang X, Huang C-J, Yu G-Z, Wang J-J, Wang R, et al. (2013) Expression of group IIA phospholipase $\mathrm{A} 2$ is an independent predictor of favorable outcome for patients with gastric cancer. Hum Pathol 44: 2020-2027. [Crossref] 
77. Davis ME, Zuckerman JE, Choi CHJ, Seligson D, Tolcher A, et al. (2010) Evidence of RNAi in humans from systemically administered siRNA via targeted nanoparticles. Nature 464: 1067-1070. [Crossref]

78. Yu JA, Kalatardi S, Dohse J, Sadaria MR, Meng X, et al. (2012) Group IIa sPLA2 Inhibition Attenuates NF- $\mathrm{kB}$ Activity and Promotes Apoptosis of Lung Cancer Cells. Anticancer Res 32: 3601-3607. [Crossref]

79. Kupert E, Anderson M, Liu Y, Succop P, Lu S (2011) Plasma secretory phospholipase A2-IIa as a potential biomarker for lung cancer in patients with solitary pulmonary nodules. Bmc Cancer 11: 513. [Crossref]

80. Graff JR, Konicek BW, Deddens JA, Chedid M, Hurst BM, et al. (2001) Expression of group IIa secretory phospholipase A2 increases with prostate tumor grade. Clin Cancer Res 7: 3857-3861. [Crossref]

81. Sarate RM, Chovatiya GL, Ravi V, Khade B, Gupta S, et al. (2016) sPLA2-IIA Overexpression in Mice Epidermis Depletes Hair Follicle Stem Cells and Induces Differentiation Mediated Through Enhanced JNK/c-Jun Activation. Stem Cells 34: 2407-2417 [Crossref]

82. Martín R, Cordova C, Gutiérrez B, Hernández M, Nieto ML (2017) A dangerous liaison: Leptin and sPLA2-IIA join forces to induce proliferation and migration of astrocytoma cells. PLoS One 12: e0170675. [Crossref]

83. Tribler L, Jensen LT, Jørgensen K, Brünner N, Gelb MH, et al. (2007) Increased expression and activity of group IIA and X secretory phospholipase A2 in peritumoral versus central colon carcinoma tissue. Anticancer Res 27: 3179-3185. [Crossref]

84. Miki Y, Kidoguchi Y, Sato M, Taketomi Y, Taya C, et al. (2016) Dual Roles of Group IID Phospholipase A2 in Inflammation and Cancer. J Biol Chem 291: 15588-15601. [Crossref]

85. Fan L, Lei H, Lin Y, Zhou Z, Shu G, et al. (2020) CXCL13, FCRLA, PLA2G2D and MS4A1 Were Identified As Prognostic Biomarkers and Correlated with Immune Infiltration in Ovarian Cancer by Integrating TMB and Microenvironment Analysis. Available at SSRN 3514638. [Crossref]

86. Igarashi A, Shibata Y, Yamauchi K, Osaka D, Takabatake N, et al. (2009) Gly80Ser Polymorphism of Phospholipase A2-IID Is Associated with Cytokine Inducibility in A549 Cells. Respiration 78: 312-321. [Crossref]

87. Murakami M, Yoshihara K, Shimbara S, Lambeau G, Gelb MH, et al. (2002) Cellular Arachidonate-releasing Function and Inflammation-associated Expression of Group IIF Secretory Phospholipase A2. J Biol Chem 277: 19145-19155. [Crossref]

88. Murase R, Taketomi Y, Miki Y, Nishito Y, Saito M, et al. (2017) Group III phospholipase A 2 promotes colitis and colorectal cancer. Sci Rep 7: 12261. [Crossref]

89. Lyu X, Jiang Y, Zhang M, Li G, Li G, et al. (2019) Genomic stratification based on radiosensitivity and PD-L1 for tailoring therapeutic strategies in cervical cancer. Epigenomics 11: 1075-1088. [Crossref]

90. Surrel F, Jemel I, Boilard E, Bollinger JG, Payre C, et al. (2009) Group X Phospholipase A2 Stimulates the Proliferation of Colon Cancer Cells by Producing Various Lipid Mediators. Mol Pharmacol 76: 778-790. [Crossref]
91. Scott GA, Jacobs SE, Pentland AP sPLA2-X Stimulates Cutaneous Melanocyte Dendricity and Pigmentation Through a Lysophosphatidylcholine-Dependent Mechanism. Journal of Investigative Dermatology 126: 855-861. [Crossref]

92. Neve RM, Chin K, Fridlyand J, Yeh J, Baehner FL, et al. (2006) A collection of breas cancer cell lines for the study of functionally distinct cancer subtypes. Cancer cell 10: 515-527. [Crossref]

93. Giordanetto F, Pettersen D, Starke I, Nordberg P, Dahlström M, et al. (2016) Discovery of AZD2716: a novel secreted phospholipase A2 (sPLA2) inhibitor for the treatment of coronary artery disease. ACS Med Chem Lett 7: 884-889. [Crossref]

94. Shaposhnik Z, Wang X, Trias J, Fraser H, Lusis AJ (2009) The synergistic inhibition of atherogenesis in apoE-1- mice between pravastatin and the sPLA2 inhibitor varespladib (A-002). Journal of lipid research 50: 623-629. [Crossref]

95. Yokota Y, Hanasaki K, Ono T, Nakazato H, Kobayashi T, et al. (1999) Suppression of murine endotoxic shock by sPLA2 inhibitor, indoxam, through group IIA sPLA2independent mechanisms. Biochim Biophys Acta 1438: 213-222. [Crossref]

96. Henderson WR Jr, Oslund RC, Bollinger JG, Ye X, Tien Y-T, et al. (2011) Blockade of human group X secreted phospholipase A2 (GX-sPLA2)-induced airway inflammation and hyperresponsiveness in a mouse asthma model by a selective GX-sPLA2 inhibitor J Biol Chem 286: 28049-28055. [Crossref]

97. Wang Q, Sun AY, Pardeike J, Müller RH, Simonyi A, et al. (2009) Neuroprotective effects of a nanocrystal formulation of sPLA2 inhibitor PX-18 in cerebral ischemia/ reperfusion in gerbils. Brain research 1285: 188-195. [Crossref]

98. Hoda MN, Singh I, Singh AK, Khan M (2009) Reduction of lipoxidative load by secretory phospholipase A2 inhibition protects against neurovascular injury following experimental stroke in rat. J Neuroinflammation 6: 21. [Crossref]

99. Jung B, Kim J, Bae J-S (2016) Dabrafenib, as a novel insight into drug repositioning against secretory group IIa phospholipase A2. Int J Immunopathol Pharmacol 12: 415 421. [Crossref]

100. Knerr L, Giordanetto F, Nordberg P, Pettersen D, Selmi N, et al. (2018) Discovery of a series of indole- 2 carboxamides as selective secreted phospholipase A2 type X (sPLA2-X) inhibitors. ACS Med Chem Lett 9: 594-599. [Crossref]

101. Dhananjaya BL, Shivalingaiah S (2016) The anti-inflammatory activity of standard aqueous stem bark extract of Mangifera indica L. as evident in inhibition of Group IA sPLA2. An Acad Bras Cienc 88: 197-209. [Crossref]

102. Zhang D, Li J, Sun S, Huang C (2018) The inhibitory effect of saPLI $\gamma$, a snake sourced PLA2 inhibitor on carrageenan-induced inflammation in mice. Toxicon 151: 89-95. [Crossref]

103. Novo Belchor M, Hessel Gaeta H, Fabri Bittencourt Rodrigues C, Ramos da Cruz Costa C, de Oliveira Toyama D, et al. (2017) Evaluation of Rhamnetin as an inhibitor of the pharmacological effect of secretory phospholipase A2. Molecules 22: 1441. [Crossref]

Copyright: (C2020 Fang-Yuan Z. This is an open-access article distributed under the terms of the Creative Commons Attribution License, which permits unrestricted use, distribution, and reproduction in any medium, provided the original author and source are credited. 